\title{
Fuzzy-Based Approach for Assessing Traffic Congestion in Urban Areas
}

\author{
Sara Berrouk ${ }^{1(\bowtie)}$, Abdelaziz El Fazziki $^{2}$, and Mohammed Sadgal ${ }^{2}$ \\ ${ }^{1}$ Faculty of Science and Technology, Cadi-Ayyad University, Marrakesh, \\ Morocco \\ berrouk.sara@gmail.com \\ 2 Faculty of Science, Cadi-Ayyad University, Marrakesh, Morocco \\ \{elfazziki, sadgal\}@uca.ma
}

\begin{abstract}
The very rapid evolution of urban areas leads to a reflection on the citizens' mobility inside the cities. This mobility problem is highlighted by the increase in terms of time, distance and social and economic costs, whereas the congestion management approach implemented rarely meets the road users' expectations. To overcome this problem, a novel approach for evaluating urban traffic congestion is proposed. Factors such as the imprecision of traffic records, the user's perception of the road's level of service provided and variation in sample data are mandatory to describe the real traffic condition. To respond to these requirements, a fuzzy inference-based method is suggested. It combines three independent congestion measures which are: speed ratio, volume to capacity ratio and decreased speed ratio into a single composite measure which is the congestion index. To run the proposed fuzzy model, the traffic dataset of Austin-Texas is used. Although it is still not possible to determine the best congestion measure, the proposed approach gives a composite aspect of traffic congestion by combining and incorporating the uncertainty of the three independent measures.
\end{abstract}

Keywords: Congestion measures $\cdot$ Fuzzy inference systems $\cdot$ Congestion estimation

\section{Introduction}

Traffic congestion, not restricted to but particularly predominant in big cities, is considered as the most prominently compounding issue related to traffic engineering and urban arranging, with clear ramifications on urban economy, environment, and way of life [1]. Traffic in urban areas keeps on developing especially in large cities of developing countries, which are portrayed by substantial monetary and population extension. This normally requires serious transportation of products and travelers, expanding interest for individual vehicular proprietorship that in the course of the most recent decade has seen exponential development around the world [2]. Catching congestion ends up significant in this regard, yet rather fundamental.

In the past few years, the traffic congestion phenomenon has been widely and differently described as a physical condition that occurs in traffic streams which leads to 
restrained movement, prolonged delays and decreased speeds [3]. Its definition is conventionally classified based on four aspects: Speed, capacity, travel time/delay and the congestion cost [4]. As a result, the level of congestion on urban roads is measured and evaluated uniformly. In this sense, a novel approach for evaluating urban traffic congestion is proposed. Since the phenomenon of congestion does not have a uniform definition, its measuring on the different road segments should not be done uniformly. A fuzzy inference-based method is suggested that incorporate the uncertainty of the independent congestion measures. It takes into consideration the variation in the speed at peak and non-peak hours, the relationship between the vehicle counts and the capacity of roadway segments as well as the ratio between the average speed and the maximum permissible speed. As a result, this study makes it possible to tackle the vague concept of congestion from multiple aspects.

The remainders of this paper are organized as follows: Sect. 2 presents the literature review. The new fuzzy-based approach and its calculation process are proposed in Sect. 3. Section 4 illustrates the experimentation and results. the conclusion is described in Sect. 5 .

\section{Literature Review}

\subsection{Traffic Congestion Evaluation Methods}

Traffic congestion is considered to be a relative measure compared to other traffic flow parameters. The traveler perception of the service provided by the transportation system at a specific time determines the presence of congestion, which requires the definition of congestion indicators [4]. As indicated in [5], the Level Of Service (LOS) is the best observational pointer of jams in the transportation system, where the proportions of supply and demand are analyzed and classified in one of six classes that range from A (free flow) to F (highly congested). There is no limit determined for LOS to portray the congestion state, however, obviously the LOS F is characterized as the most noticeably terrible condition of stream and speaks to blocked stream. Even though a few reports are utilizing other levels of services ( $\mathrm{D}$ and $\mathrm{E}$ ) as a congested stream, LOS F is commonly acknowledged as a condition of traffic stream and hence LOS is the most fitting congestion indicator. [6] provides a review of the congestion measures, the three regularly utilized indicators of traffic congestion are examined in the following paragraphs.

The roadway congestion index (RCI), first introduced by David Schrank in [7], is an indicator of the congestion severity in a wide area. The RCI compares between the daily vehicle miles per lane-mile (DVMT) of an area and the total predicted vehicle miles in congested conditions in that specific area, both measured depending on the road type (freeway or arterial roadway), Eq. 1 shows how the RCI is computed:

$$
\mathrm{RCI}=\frac{\left(\begin{array}{c}
\text { (Freeway DVMT }) \times(\text { Freeway Daily_vehicule_mile }) \\
(\text { Arterial DVMT }) \times(\text { Arterial Daily_vehicule_mile })
\end{array}\right.}{\left(\begin{array}{c}
13,000 \times \text { Freeway Daily_vehicule_mile } \\
+5,000 \times \text { Arterial Daily_vehicule_mile }
\end{array}\right)}
$$


The RCI accepts a capacity limit of 13,000 DVMT on freeways and 5,000 DVMT on head arterials. An RCI estimation of 1.0 or more reflects a bothersome congestion level. But the fact that it's hard for road users to relate their travel experience to this index can be considered as a drawback because it infects the ability to forecast future conditions. This indicator is incapable to infer the vital traffic improvement plans to avoid traffic congestion since it is an area-wide measure.

The Relative Delay Rate (RDR) and the Congestion Index (CI) are two well-used congestion indicators, introduced respectively by Lomax in [4] and Taylor in [8]. They both translate the road users' perception of the traffic flow quality relatively to a perfect or tolerable condition. The RDR is calculated using Eq. 2:

$$
\underset{\text { Relative }}{\text { Delay Rate }}=\frac{\left[\begin{array}{c}
\text { Actual travel rate }(\text { min per mile }) \\
- \text { Tolerable travel rate }(\text { min per mile })
\end{array}\right]}{\text { Tolerable travel rate }(\text { min per mile })}
$$

where the travel rate is the travel time per segment length. The congestion index is calculated using the Eq. 3:

$$
\text { Congestion index }=\frac{\left(\begin{array}{c}
\text { Actual travel } \\
\text { time of section }
\end{array}-\begin{array}{c}
\text { FreeFlow } \\
\text { travel time }
\end{array}\right)}{\text { FreeFlow travel time }}
$$

Where the free-flow travel time represents the time spent traveling at the mentioned speed limit. An estimation of zero of the congestion index shows a low degree of congestion and considered to be near free-flow condition. An estimation higher than 2 of Eq. (3) reflects a severely congested condition. Most of the time, the use of the congestion index can be beneficial when traffic conditions are compared on several roadways since the records can be collected independently for various road segments.

\subsection{Limitations in Traffic Congestion Assessment Methods}

In this section, the limitations in assessing urban traffic congestion are mentioned. First, the imprecision of traffic data records and observations gathered in both the supply and demand sides. In the supply-side parameters, a single value describing the traffic state can be misleading since the traffic signal timing and geometric design parameters changes according to the road section and the study period. For the demand-side, parameters such as travel time, vehicle counts and delay can also be imprecise. We can conclude that uncertainty is associated with all congestion measures no matter how accurate their representation of the actual traffic state is. Second, the notion of acceptable or tolerable traffic conditions is fuzzy because it differs according to the roadway section and the road user's experience. Therefore, it is impossible to use a unique value to describe it. Third, to measure the traffic congestion, stepwise measures can be used such as the Level Of Service (LOS). These measures can be misleading in case the traffic situation is close to a threshold because often the situation will exceed 
the latter. This problem can be touched when the studied road section knows constantly changing geometric features and traffic parameters. Fourth, measures of congestion in the literature are mainly related to single traffic parameters; often it is travel speed or delay. However, road congestion is a vague concept, and the traveler impression to describe it is influenced by many factors. In this sense, a combination of more than one measure to evaluate the traffic condition is required. Fifth, the road user's opinion of what is tolerable or intolerable traffic condition should be incorporated when measuring road congestion. This boundary is not clear because of the opinion, the travel condition and between localities differ among travelers. Thus, an ideal measure should take into consideration the flexibility that represents the conditions of the locality.

To sum up, traffic congestion is a complex phenomenon that can be described in different manners and caused by multiple factors. Therefore, it is necessary to take into account the imprecise quantities and the traveler's perception in the process of assessing the level of congestion. Considering the problems aforementioned, in this paper, a fuzzy-based method is proposed to determine the level of traffic congestion. It is believed that the proposed method can take into consideration the following requirements of an ideal congestion measuring approach:

1. Integration of the imprecise nature of traffic data,

2. Integration of the subjective notion of travelers perceptions of traffic congestion (acceptable or inacceptable service quality),

3. And finally, the combination of multiple measures to form a composite measure.

\section{A Congestion Measure Based on the Fuzzy Inference System}

The proposed fuzzy process, illustrated in Fig. 1, is used to measure the congestion levels by using traffic data (such as vehicle counts, speed, etc.). This process provides a solution for ambiguous and uncertain problems. The procedure involves the calculation of the values of the input parameters, the categorization of the input parameters values into distinguishing groups, the definition of the various congestion states, and the determination of the congestion index values.

\subsection{Data Acquisition and Preparation}

To validate our proposal, the choice fell on the Radar traffic Counts [9] and Travel Sensor [10] datasets provided by the transportation department of Austin City. The choice of these datasets was not arbitrary: the dataset offers traffic parameters needed to conduct our study (such as volume, speed, etc.) and they put the light on the intersections in Austin having a heavy traffic flow which will reflect the benefits of our proposed system in detecting the most congested intersections and road segments. The output of the system can be so beneficial in the making of short and long terms decisions from the authorities. The sensors cover a total of 17 intersections, each having a specific number of lanes which made it possible to analyze the congestion in about 76 road segments. The traffic count and speed data are gathered from a specific 


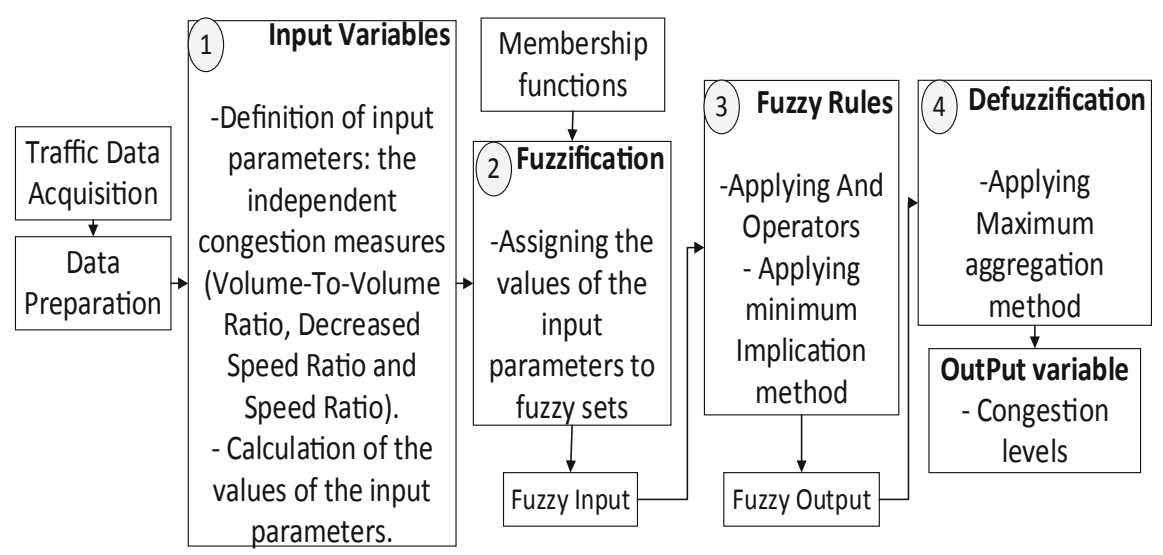

Fig. 1. The proposed fuzzy logic process for congestion evaluation

type of sensors deployed by the city of Austin which are the Wavetronix radar sensors. These data have been collected since the creation of the dataset in November 2017 with an interval of $15 \mathrm{~min}$; which led to a huge amount of data (about $4.65 \mathrm{M}$ records). In this study, one month of data (from 01-March-2019 to 31-March-2019) is used to test the proposed system and minimize its running time. The peak and non-peak speed data are extracted from the dataset considering that the morning peak-hours extend from 9:30 am to $11: 30 \mathrm{am}$, the evening peak hours extend from 5:30 pm to 7:30 pm and the non-peak hours extend from 7:00 am to 9:00 am and from 12:00 am to 5:00 pm. Other data was added to the dataset to fulfill the need of this study such as the capacity and the maximum permissible speed of each segment.

\subsection{Input Parameters}

The three measures are used to describe congestion, which are: volume-to-capacity ratio, the decreased speed ratio, and the speed ratio. The Volume to capacity ratio measure (Eq. 4) reflects the ratio between the counts of vehicles passing through a road segment and the maximum number of vehicles that that road segment can support. This value ranges from 0 to values sometimes greater than 1 . A value near to " 0 " represents the best traffic state (free flow), and a value near or greater than 1 represents the worst traffic state (severely congested).

$$
\mathrm{V} / \mathrm{C} \text { Ratio }=\frac{\text { volume of vehicles }}{\text { capacity of road segment }}
$$

The second measure is the decreased speed ratio (Eq. 5) that denotes the rate of reduced speed of the vehicle due to a congested situation. This measure represents the traffic condition of the road network for non-peak and peak periods. This value ranges from 0 to 1,0 being the best condition when the Peak average speed is bigger than or 
equal to the Non-Peak average speed, and 1 being the worst condition when Peak's average speed is near 0 .

$$
\text { Decreased speed ratio }=\frac{\text { NonPeakAvgSpeed }- \text { PeakAvgSpeed }}{\text { NonPeakAvgSpeed }}
$$

The third measure is the speed ratio (Eq. 6); it reflects the ratio between the average speed and the maximum allowed speed on a road segment. It is used as an evaluation indicator of the traffic state in urban areas. The speed ratio value ranges from 0 to 1 , three threshold values $(0.25,0.5$, and 0.75$)$ are adopted to be the classification criterion of the traffic condition. The extreme values 0 and 1 reflect the worst and the best conditions of traffic respectively.

$$
\text { Speed Ratio }=\frac{\text { AvgSpeed }}{\text { SpeedMax }}
$$

\subsection{Fuzzification Phase}

After computing the congestion measures, a domain transformation called fuzzification is launched. This process presents the first stage of the fuzzy logic processing. It consists of assigning the values to fuzzy sets that have vague boundaries. The fuzzy sets are classes of road conditions, simplified by a natural language such as "moderate congestion". The values of the volume-to-capacity ratio are grouped into three classes (Low, Moderate and High). Figure. 2 shows the given values of the v/c ratio and their mapping to the three classes with the help of the membership functions.

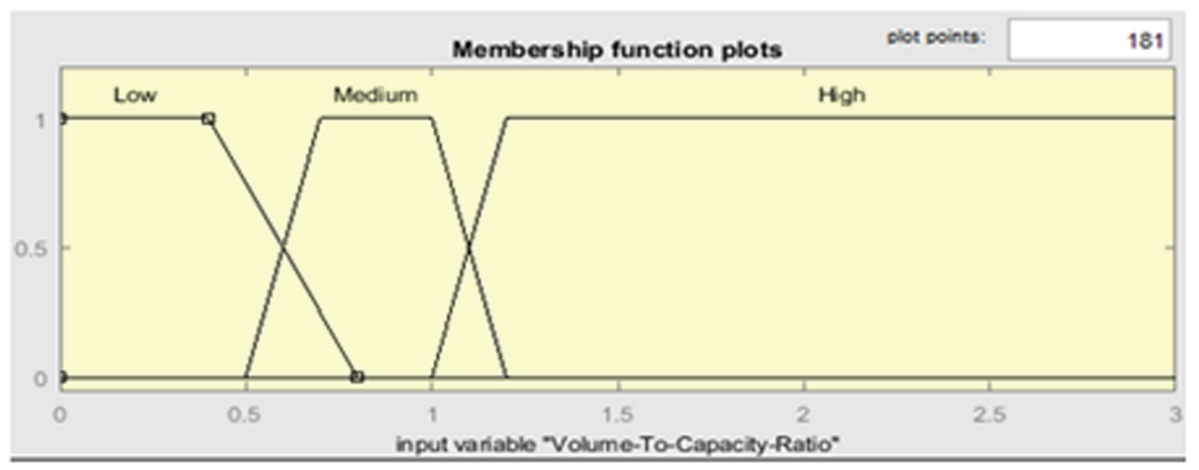

Fig. 2. Membership function for volume to capacity ratio

For the decreased speed ratio measure, the values are categorized into six comprehensive categories by the traveler. The six categories range from $\mathrm{A}$ to $\mathrm{F}$ and the defined membership functions for each one of the six classes in which the value of the decreased speed ratio is given in Fig. 3 . 


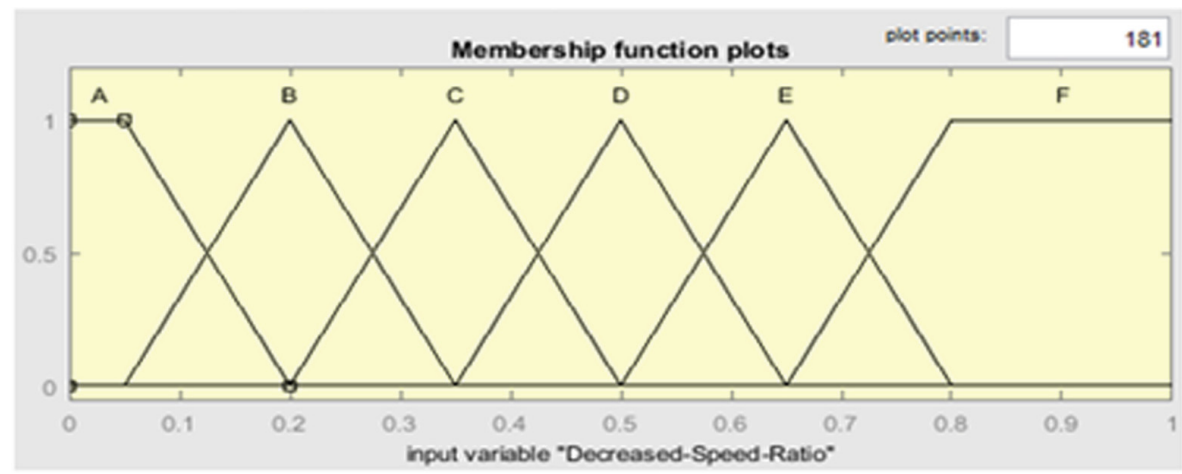

Fig. 3. Membership function for the decreased speed ratio

In like manner, four classes are identified to classify the values of the speed Ratio. The classes are Free Flow, Moderate, High and Very High. Figure 4 shows the membership functions of the four classes in which the value of the speed ratio is given and the compatibility of this value with a specific class.

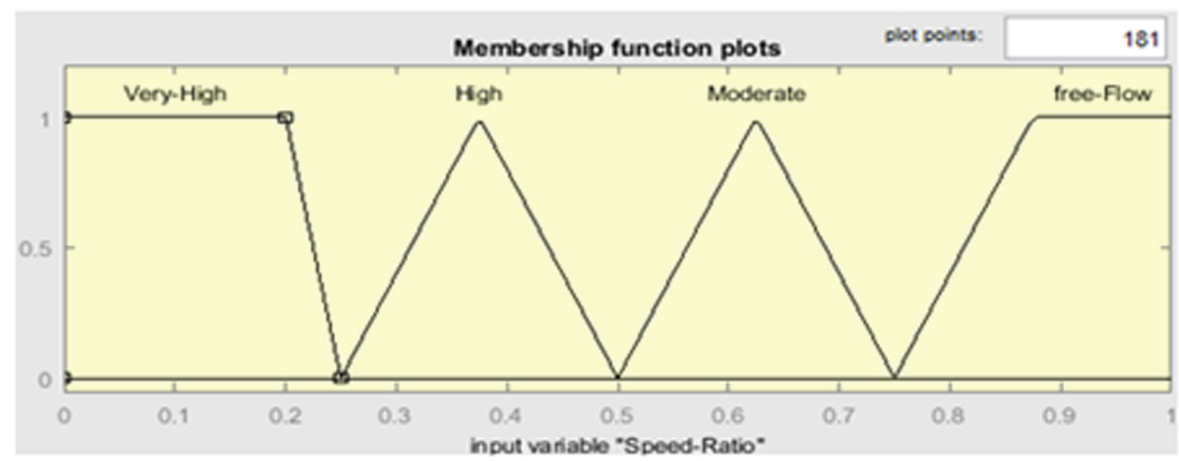

Fig. 4. Membership function for speed ratio

\subsection{Defuzzification Phase}

In this stage, the fuzzy output is converted to a number. In this study, the output of the fuzzy process is the congestion index. It is classified into four groups: "smooth", "mild congested", "congested" and "heavily congested". It ranges from 0 to 1 , with 0 reflecting the best traffic condition and 1 reflects the worst. Figure 5 shows the membership function of the congestion index. The four congestion index classes are defined on the scale from 0 to 1 and each one is assigned to a natural comprehensible language term (see Fig. 5). 


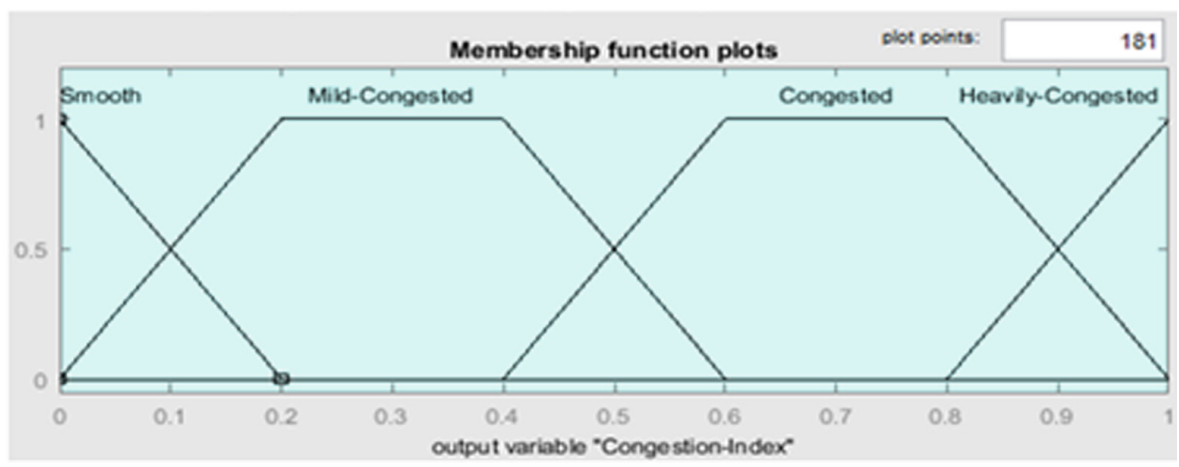

Fig. 5. Membership function for the proposed congestion index

\subsection{Fuzzy Rule Base}

Numerous fuzzy rules are performed to combine the three input parameters and to obtain a natural-language and comprehensive congestion measure. The fuzzy rules contain two parts. The first part is the antecedent; it is the one following "IF". And the second part is the consequent; it is the one following "Then". The fuzzy rules for each transport mode are described in the following way:

Rule: IF Volume-To-Capacity Ratio is A And Decreased Speed Ratio is B And Speed Ratio is $\mathrm{C}$ Then the congestion level is $\mathrm{D}$.

Where A, B, C, and D each denote the level of congestion as mentioned in Figs. 2, 3,4 and 5 .

\section{Experimentation and Results}

\subsection{Congestion Index Computing: Application of FIS}

In this paper, MATLAB Toolbox [11] for fuzzy modeling is used to estimate the level of traffic congestion. The proposed fuzzy architecture is designed with three congestion measures as inputs, congestion index as the only output and seventy-two fuzzy rules. Having specific estimations of the volume-to-capacity ratio, the decreased speed ratio and the speed ratio as inputs for the rules, a match is done by a particular rule between the input values and the antecedent of the rule using the aforementioned membership functions (Figs. 2, 3 and 4). Doing so, the minimum estimation of the membership function levels among the three is considered as the truth of the rule's antecedent. Furthermore, this estimation affects the truth of the rule's consequent. In the case where the values of the inputs belong to more than a class, all possible rules can be either used or fired and the result is formed by combining the consequents of the possible. How the rules are executed and the result is computed is shown in Fig. 6. 

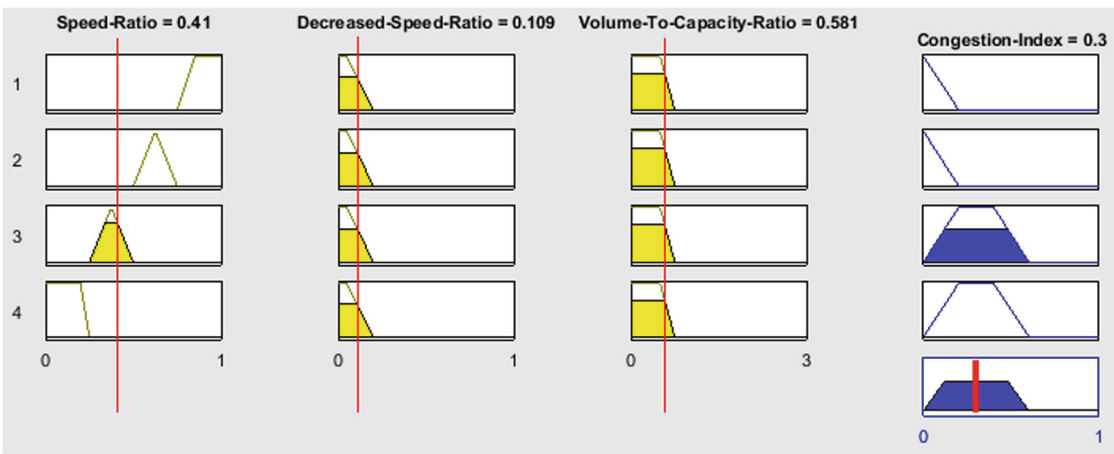

Fig. 6. The calculation of the congestion index using the proposed fuzzy model

\subsection{Results and Discussion}

Table 1 shows an example of the congestion index generation for some segments of the study area. It may be observed that using either one of the input parameters cannot give a real picture of the traffic congestion. The combination of all three parameters captures the real status of the condition. It is observed that if one of the input parameters is much higher and the remaining two parameters are on the lower side as is the case of segment 10, the congestion effect is moderate. However, on the traffic link 23, all the three parameters are of the noticeable amount resulting higher congestion effect. Similarly, the links subjected to lower values of speed reduction rate, very low-speed rate and v/c ratio resulting in lower congestion index such as segment 40. The proposed model gives a more realistic and detailed congestion picture compared to that obtained in traditional methods by considering a single parameter.

Table 1. The caption of the generated congestion index for some road segments

\begin{tabular}{l|l|l|l|l|l|l|l|l}
\hline $\begin{array}{l}\text { Segment } \\
\text { number }\end{array}$ & $\begin{array}{l}\text { Vehicle } \\
\text { counts }\end{array}$ & $\begin{array}{l}\text { Average } \\
\text { speed }\end{array}$ & $\begin{array}{l}\text { Non-peak } \\
\text { average } \\
\text { speed }\end{array}$ & $\begin{array}{l}\text { Peak } \\
\text { average } \\
\text { speed }\end{array}$ & $\begin{array}{l}\text { Volume to } \\
\text { capacity } \\
\text { ratio }\end{array}$ & $\begin{array}{l}\text { Decreased } \\
\text { speed ratio }\end{array}$ & $\begin{array}{l}\text { Speed } \\
\text { ratio }\end{array}$ & $\begin{array}{l}\text { Resulting } \\
\text { congestion } \\
\text { index }\end{array}$ \\
\hline 3 & 69.35 & 34.48 & 36.5 & 24.25 & 0.73 & 0.33 & 0.86 & 0.299 \\
\hline 5 & 72.73 & 26.69 & 37.2 & 16.7 & 1.72 & 0.55 & 0.67 & 0.7 \\
\hline 10 & 70.89 & 32.20 & 31.04 & 13.5 & 1.13 & 0.56 & 0.81 & 0.54 \\
\hline 17 & 111.71 & 32.08 & 27.2 & 15.4 & 1.04 & 0.44 & 0.802 & 0.41 \\
\hline 23 & 75.78 & 33.4 & 37.5 & 5.02 & 2.05 & 0.86 & 0.74 & 0.906 \\
\hline 40 & 103.16 & 42.07 & 40.25 & 22.37 & 0.95 & 0.44 & 0.84 & 0.3 \\
\hline 69 & 89.08 & 22.14 & 33.5 & 17.42 & 2.07 & 0.92 & 0.4 & 0.934 \\
\hline 71 & 68.15 & 37.93 & 42.17 & 29.37 & 1.74 & 0.43 & 0.68 & 0.7 \\
\hline
\end{tabular}




\section{Conclusion}

The contribution in this paper consists of modeling a fuzzy-based method for urban traffic congestion measuring. It combines three inputs which are traditionally used as measures of traffic congestion into one output which is a composite congestion measure. Each one of the independent measures, the speed ratio, the volume to capacity ratio and the decreased speed ratio, describes a particular aspect of the traffic conditions using precise thresholds. Doing so, the error possibility in measurement is high which misleads the representation of the real traffic condition. The suggested fuzzy inference approach takes into consideration every little variation in the input congestion measures and their combination provides a more accurate representation of the traffic state. The accuracy of such a model is higher because it is based on natural-language rules which reflect perfectly the traveler perception of the traffic condition. The suggested method can be used to evaluate the congestion over road sections, arterials, or highway road network. In terms of perspectives, we are attempting to expand the proposed model to incorporate other factors playing a considerable role in changing the traffic condition such as parking in streets, roads structure, etc. Further work will make possible to redefine the membership functions and the rules more precisely.

\section{References}

1. Wang, H., et al.: Joint link-based credit charging and road capacity improvement in continuous network design problem. Transp. Res. Part A: Policy Pract. 67, 1-14 (2014)

2. Li, Z., Huang, J.: How to mitigate traffic congestion based on improved ant colony algorithm : a case study of a congested old area of a metropolis. Sustainability 11(4), 1140 (2019)

3. Weisbrod, G., Vary, D., Treyz, G.: Economic Implications of Congestion, Washington, DC (2001)

4. Lomax, T., et. al.: Quantifying congestion. NCHRP Report 398, Transportation Research Board 1 and 2, Washington DC (1997)

5. Cottrell, B.W.D.: Empirical freeway queuing duration model. J. Transp. Eng. 127(1), 13-20 (2001)

6. Rao, K.R., Rao, A.M.: Measuring urban traffic congestion - a review. Int. J. Traffic Transp. Eng. 2(4), 286-305 (2012)

7. Schrank, D.L., Turner, S.M., Lomax, T.J.: Estimates of Urban Roadway Congestion - 1999, vol. 1, no. 2. Texas A\&M University, College Station (1993)

8. Taylor, M.A.P.: Exploring the nature of urban traffic congestion: concepts, parameters, theories and models. In: 16th Meeting of the Australian Road Research Board, p. 16 (1992)

9. Radar Traffic Counts Dataset of Austin-Texas city (2016). https://data.austintexas.gov/ Transportation-and-Mobility/Radar-Traffic-Counts/1626-g7ub Accessed 01 Oct 2019

10. Travel Sensor Dataset of Austin-Texas City (2016). https://data.austintexas.gov/ Transportation-and-Mobility/Travel-Sensors/6yd9-yz29 Accessed 01 Oct 2019

11. Matlab Toolbox. https://www.mathworks.com/help/thingspeak/matlab-toolbox-access.html Accessed 20 March 2020 